\title{
COVID-19 Specific Risk Markers for Intimate Partner Violence Perpetration
}

\author{
Chelsea M. Spencer ${ }^{1}\left[\right.$ Caroline Gimarc $^{1} \cdot$ Jared Durtschi $^{1}$
}

Accepted: 12 October 2021 / Published online: 19 October 2021

(c) The Author(s), under exclusive licence to Springer Science+Business Media, LLC, part of Springer Nature 2021

\begin{abstract}
Intimate partner violence (IPV) rates have grown alongside the sweeping changes, challenges, and transitions necessitated by the onset of COVID-19. The goal of this exploratory study was to examine COVID-19 related risk markers for IPV perpetration. Data were collected from a national sample of 365 U.S. individuals who were in a relationship during August 2020, in the midst of the COVID-19 epidemic. Unadjusted odds ratios were calculated for 27 unique risk markers related to lifestyle changes due to COVID-19, mental health, isolation, financial impacts, and COVID-19 diagnoses. The strongest risk markers for IPV perpetration were feelings of loneliness, followed by anxiety symptoms, perceived stress, fear, boredom, substance use and lifestyle changes. Understanding risk markers associated with an increase in IPV perpetration can aid helping professionals identify individuals who may be at risk for IPV, or target these factors to aid in IPV prevention and intervention efforts.
\end{abstract}

Keywords COVID-19 $\cdot$ Intimate partner violence $\cdot$ Risk assessment $\cdot$ Perpetration

Intimate partner violence (IPV) is a large problem in the United States (US), with approximately $32 \%$ of women and $28 \%$ of men experiencing physical IPV, $16.4 \%$ of women and $7 \%$ of men experiencing sexual IPV, and $47 \%$ of both women and men experiencing psychological IPV in their lifetime (Smith et al., 2017). IPV victimization has been associated with a wide range of short-term and long-term negative impacts, such as physical health consequences, negative mental health symptoms (e.g., depressive symptoms, anxiety, posttraumatic stress symptoms), substance use, fear, and missed school or work (Breiding et al., 2014; Campbell, 2002; Coker et al., 2000; Wong \& Mellor, 2014). Reducing the prevalence of IPV is an important societal goal, and examining factors associated with IPV perpetration can aid in assessment and intervention efforts in hopes of reducing instances of IPV.

During the COVID-19 pandemic, there has been heightened awareness and concern about increases in cases of IPV (Moreira \& da Costa, 2020; Peterman et al., 2020; World Health Organization [WHO], 2020a). Stay at home orders, movement restrictions, economic uncertainty, social isolation, and other additional stressors plaguing families can cause increased conflict among couples, which can increase instances of IPV (Peterman et al., 2020; van Gelder et al., 2020; WHO, 2020a). Preliminary data in the US compared the number of calls and arrests related to family violence in March 2020 versus March 2019 and found that certain departments have reported an $18 \%-24 \%$ increase in calls (City of San Antonio, 2020; Jefferson County Sherriff's Office, 2020). Additionally, we know that not all instances of IPV are reported to law enforcement, and victims of IPV may be reluctant to seek outside resources during the pandemic (Kaukinen, 2020), which may suggest that the increased rates of IPV are far greater than law enforcement data. Due to the increased attention and concern related to an increase in cases of IPV since the start of the COVID-19 pandemic, the goal of this exploratory research is to examine risk markers for IPV perpetration related to the COVID-19 pandemic to aid in assessment, identification, and intervention efforts.

Chelsea M. Spencer

cspencer@ksu.edu

1 Kansas State University, Manhattan, KS, USA 


\section{COVID-19 and Risk for IPV Perpetration}

\section{COVID-19, Isolation and Mental Health}

There has been a large concern about the mental health ramifications associated with the COVID-19 pandemic (WHO, 2020b). In particular, there has been considerable concern about how the social isolation associated with prolonged periods of quarantine may increase negative mental health symptoms, such as depressive symptoms, anxiety, substance use, loneliness, or suicidal ideation (WHO, 2020c). One study that surveyed 1210 individuals in China found that $53 \%$ of the sample reported a moderate to severe psychological impact related to the COVID19 pandemic (Wang et al., 2020). Another recent survey found that approximately 16-28\% of individuals report depressive symptoms or anxiety as a reaction to the pandemic (Rajkumar, 2020). Additionally, fear of COVID-19 was associated with both depressive and anxiety symptoms in the US (Fitzpatrick et al., 2020). It is also known that mental health issues, such as depression, anxiety, posttraumatic stress, substance use, and borderline personality disorder are all risk markers for IPV perpetration (Author et al., 2019; Cafferky et al., 2018; Oram et al., 2014). Additionally, social isolation has been associated with IPV as a tactic perpetrators use to control their victims (James et al., 2004). Therefore, in this study we examined various mental health and isolation factors, as they were reported during the COVID-19 pandemic, as potential risk markers for IPV perpetration.

\section{COVID-19 and Lifestyle Changes}

Undoubtedly, the COVID-19 pandemic has had a large impact on nearly everyone's day-to-day living conditions and lifestyle. In order to help slow the spread of COVID19 , many are in quarantine, working from home, social distancing, wearing face masks, and not attending large gatherings. In addition, a study in Canada found that $74 \%$ of mothers and $87 \%$ of fathers reported increased screen time since the pandemic (Carroll et al., 2020). It was also found that increased media exposure related to COVID19 (frequency, duration, and different media platforms) were significantly related to higher levels of depression and anxiety (Bendau et al., 2020). There is a potential of negative consequences faced by individuals who have had lifestyle changes related to COVID-19, and thus we examined them as potential risk markers for IPV perpetration.

\section{COVID-19 and Financial Stress}

Financial stress, unemployment, and employment insecurity have all been consequences for many individuals during the COVID-19 pandemic (International Labor Organization, 2020). In the US, it was reported that in April 2020, approximately $15 \%$ of the US population had filed for unemployment (Bureau of Labor Statistics, 2020). A qualitative study examining family stressors experienced during COVID-19 found financial insecurity as a theme, with participants reporting having to shut down their businesses or a family member losing their job as a significant source of stress (Carroll et al., 2020). Experiencing financial stress or unemployment can have severe negative mental outcomes, physical outcomes, and negative relationship outcomes (Dakin \& Wampler, 2008; McKeeRyan et al., 2005). For example, financial stress is related to increased hostile interactions and arguments centered around finances (Britt et al., 2010; Conger et al., 1990). Additionally, a recent meta-analysis on risk markers for IPV perpetration found that financial stress has been linked to IPV perpetration for both men and women (Author et al., 2020). Due to the immense impact that the COVID19 pandemic has had on families' financial instability and unemployment rates, we examined IPV risk markers related to financial stress and work-related impacts.

\section{COVID-19 Diagnosis}

A diagnosis of COVID-19 has tremendous potential to have large physical health consequences, including death. Commonly reported symptoms of a COVID-19 diagnosis include cough, fever, fatigue, joint/muscle pain, shortness of breath, confusion, and gastrointestinal issues (Docherty et al., 2020). Research has also noted prolonged symptoms even after one recovers from a COVID-19 diagnosis, such as poor quality of life, fatigue, chest pain, joint pain, and difficulty breathing (Carfì et al., 2020). Individuals experiencing poor physical health symptoms can experience negative mental health consequences, work ability impacts, and social isolation/rejection (Crandall \& Moriarty, 1995; Egede, 2007; Schultz et al., 2009). Additionally, fear of contracting COVID-19 is associated with anxiety and depressive symptoms (Fitzpatrick et al., 2020), so knowing someone who has received a COVID-19 diagnosis may incite fear, and thus negative mental health symptoms. Therefore, in this study we examined receiving a COVID-19 diagnosis or knowing someone who has had a positive diagnosis as potential risk markers for IPV perpetration. 


\section{Theoretical Framework}

The theoretical framework that has guided this study was Boss et al. (2016) adaptation of Hill's (1958) ABC-X model of family stress. Undoubtedly, the COVID-19 pandemic has increased stress for many families, couples, and individuals in the US, making this a strong guiding framework for this study. This model highlights how (A) an event or stressor, (B) resources, and $(C)$ perception of the event or stressor all interact with one another to determine the family's, couple's, or individual's the perception of the stressor (X). Research has confirmed that IPV perpetration is positively related to perceived levels of stress (Author et al., 2020), and using this framework, we can view IPV perpetration as a potential outcome or consequence of stress associated with the event/stressor, resources, and perceptions of the stress associated with the COVID-19 pandemic.

Although it is important to note that IPV could have occurred prior to the COVID-19 pandemic, there is also potential that stress associated with the pandemic will exacerbate the likelihood of resorting to violence in an intimate relationship. In this study, we examined stressors and resources (or lack of resources, such as financial changes, or lack of social support), and potential outcomes related to stressors (mental health problems) that have been found to be related to the COVID-19 pandemic in previous research.

\section{Current Study}

There has been serious concern about the increase in IPV since the beginning of the COVID-19 pandemic related to social isolation, mental health concerns, fear, and stressors experienced by families, such as financial stress or unhelpful lifestyle changes. To our knowledge, there has not been a study that directly examined risk markers for IPV perpetration associated with COVID-19. In this exploratory study, we look at data collected during the midst of the COVID-19 pandemic in the US and hypothesize that greater isolation, mental health challenges, financial stress/work stress, more lifestyle changes, and COVID-19 diagnoses will be associated with higher expected odds of IPV perpetration.

\section{Method}

\section{Procedures}

Participants were gathered using an electronic Qualtrics survey posted to the Prolific website. Prolific is a research tool that has tens of thousands of potential research subjects willing to take surveys for a nominal payment. In order to be eligible to participate in the survey, participants had to live in the US. Prolific facilitated that recruitment of subjects who were approximately nationally representative by gender, race, and age for our study, increasing the generalizability of our findings from these data. Participants were paid approximately \$4 USD as monetary compensation for completing the short survey. Data were collected roughly five months after the COVID-19 pandemic began, in August of 2020, to focus on the overall wellbeing of participants in the midst of the pandemic. Participants averaged approximately $20 \mathrm{~min}$ to complete the survey and were asked questions pertaining to their beliefs about COVID-19, mental health outcomes, wellbeing outcomes, and relational outcomes. Data were collected from 603 total participants. The sample was then narrowed down to participants that identified being in a relationship, leaving the final sample of 365 participants as the operational sample for this study.

\section{Participants}

The final sample included 365 individuals who reported being in a relationship at the time data were collected. The age of participants ranged from 19 to 78 years old, with the mean age being 45.77 years old $(S D=15.31)$. Gender was split evenly between men (49.3\%) and women (49.3\%), with the remaining $1.4 \%$ occupied by transmen and nonbinary individuals. More than half of the participants had a 4-year college degree or higher (62.2\%), with a large portion having an income between $\$ 10,000$ to $\$ 49,999$ (31.0\%) and $\$ 50,000$ to $\$ 99,999$ (33.7\%). The majority of the sample identified as White (75.6\%), followed by Black (9.3\%), Asian (7.7\%), Latinx (3.6\%), and 3.3\% of the sample identified as multiracial. Most participants identified as either agnostic/atheist (33.2\%) or Protestant/Christian $(31.3 \%)$. The majority of the sample stated that they were married to their partner whom they were answering questions about (68.4\%). A total of $9.3 \%$ of the sample reported engaging in IPV perpetration, either physically, sexually, or emotionally. More specifically, $8.49 \%$ reported perpetrating emotional IPV, $1.37 \%$ perpetrated sexual IPV, and $1.92 \%$ perpetrated physical IPV. For the sample, $4.7 \%$ reported testing positive for COVID-19, and on a scale of 1-7, participants reported on average a 4.38 score of fear of getting COVID-19 or getting it again if they had already had it. Lastly, $30.1 \%$ of participants reported working from home now, $6.3 \%$ reported being laid off (4.9\% furloughed), $14.8 \%$ reported working less hours, and $4.4 \%$ reported working more hours since the pandemic began.

\section{Measures}

\section{Intimate Partner Violence Perpetration}

IPV perpetration was assessed using the adapted Universal Violence Prevention Screening Protocol (Dutton et al., 
1996). Participants were asked, within the past year, if they had engaged in IPV-related activities. This tool assessed for three kinds of IPV-related behaviors: physical (e.g., "have you slapped, kicked, pushed, choked, or punched your partner?"), sexual (e.g., "have you forced or coerced your partner to have sex?"), and emotional perpetration (e.g., "have you repeatedly used words, yelled, or screamed in a way that frightened your partner, threatened your partner, put your partner down, or made your partner feel rejected?"). Participants were asked which of the behaviors they had engaged in. The responses were then recoded into engaged in IPV perpetration, including physical, sexual, and/or emotional perpetration (coded as 1) and did not engage in IPV perpetration (coded as 0$)$.

\section{Mental Health Measures}

Loneliness Feelings of loneliness were assessed using the UCLA Three-Item Loneliness Scale (Hughes et al., 2004). The three questions ranged on a three-point Likert scale and asked participants to think of themselves during the pandemic to assess, "how often do [they] feel they lack companionship," "how often do [they] feel left out," and "how often do they feel isolated from others." The scores from these three questions were then averaged and had an acceptable alpha of .83. Anxiety symptoms were assessed using the Generalized Anxiety Disorder-7 questionnaire (Spitzer et al., 2006). This measure had seven questions assessed on a fourpoint Likert scale. Participants were asked questions such as "Over the last 2 weeks, how often have you felt ...nervous, anxious or on edge?" and "...being so restless that it's hard to sit still." This scale had an acceptable alpha of .93. The scores were calculated by finding the average for each participant. Perceived stress was assessed using the Perceived Stress Scale (Cohen et al., 1983). This was a four-item measure where participants were asked to think about how often they felt equipped or unequipped to handle events in the past month. This was assessed on a five-point Likert scale. The alpha was .76 which is acceptable given the few number of questions this measure contained. Scores were then averaged to create an overall perceived stress score.

Feelings of Fear Fear was assessed using adapted questions from the American Fears Survey (Chapman University, 2018). Seven questions on a four-point Likert scale were averaged to create a total fear score. Questions asked participants how afraid they were of "dying" and "being unemployed." When combined, this scale had an acceptable alpha of .86 .

Feelings of Boredom Feelings of boredom were assessed using the Short Boredom Proneness Scale (Struk et al., 2017). Participants responded to questions about how they felt on an average day during the pandemic and were asked to what degree they agreed with statements such as "It takes more stimulation to get me going than most," and "I find it hard to entertain myself." These five questions were assessed on a five-point Likert scale and had an acceptable alpha of .87 when averaged together.

Substance Use Substance use was assessed using the National Institute on Drug Abuse Quick Screen (NIDA, 2012). Participants were asked about the frequency during the past year of the use of alcohol, tobacco products, prescription drugs for non-medical reasons, and illegal drugs. Participants could indicate the extent to which they used the substances from never to daily or almost daily on a five-point Likert scale. The alpha was .67, indicating an acceptable alpha for the four questions.

Depressive Symptoms Depressive symptoms were assessed using the Major Depressive Inventory (Olsen et al., 2003). This measure consisted of twelve questions on a six-point Likert scale. The participants were asked about the frequency of depressive symptoms they may have experienced during the past two weeks. This measure had an acceptable alpha of .94 .

Perceived Well-Being Perceived well-being was assessed using the Office for National Statistics' (ONS) subjective well-being questions (Benson et al., 2019). Participants were asked to rate, on a scale of 0 to 10 , how satisfied they were with their life, how happy they felt yesterday, how anxious they felt yesterday, and how worthwhile they felt their life was. These four questions were averaged for a total score from 0 to 10 and had an acceptable alpha of .79.

Hopefulness Feelings of hopefulness were assessed using the Adult Hope Scale (Snyder et al., 1991). This measure consisted of twelve items assessed on an eight-point Likert scale where participants were asked to think about different characteristics as they related to the participant ranging from "definitely false" to "definitely true." Participants were asked questions such as, "I can think of many ways to get myself out of a jam," and "even when others get discouraged, I know I can find a way to solve the problem." The questions were averaged to create a final score and had an acceptable alpha of .86 .

Suicidal Ideation Suicidal ideation was assessed using the Suicide Behaviors Questionnaire-Revised (Osman et al., 2001). Participants were asked if they had ever had suicidal thoughts. This variable was dichotomized where everyone who had a suicidal thought was labeled with a 1 and those who had not were labeled with a 0 . 
Fear Fear of getting COVID-19 was assessed by asking participants "How fearful are you of getting COVID-19? If you have had COVID-19, how fearful are you of getting it again?" This was assessed on a 7-point Likert scale ranging from "not fearful at all" to "moderately fearful" to "very fearful".

\section{Isolation Measures}

Amount of time spent interacting with friends/family virtually was assessed by asking how many times per week the participants talked to friends and family via phone call or texting and how many times per week participants interacted with friends and family via video chat or video conferencing. Responses were given on an eight-point scale ranging from "never or rarely" to 13 or more times a week." These two questions were then averaged. Amount of time spent interacting with friends/family in person was assessed very similarly, by asking how many times per week participants saw friends and family members face-to-face and also on an eight-point scale. To assess frequency of going outside for more than $15 \mathrm{~min}$, participants were asked to rate how often they go outside in one week on a five-point scale ranging from "never" to "7 or more times."

\section{Financial Stress Measures}

To assess whether individuals were struggling with money, participants were asked if in the past three months they had more than enough money left, some money left, just enough money left, were somewhat short of money, or were very short of money. Participants were also asked whether they have had access to the same financial resources since the COVID-19 pandemic began. This variable was assessed dichotomously.

Working less hours, working more hours, have been furloughed, have been fired since mid-March 2020 were all assessed by asking participants to check off whether or not they had experienced these changes in their employment.

\section{Lifestyle Changes Measures}

Working from home was assessed by asking participants to indicate whether or not they were now working from home as a result of the pandemic. Time spent on social media and reading/watching the news were measured by asking how much time, in hours, participant spent on these activities on an average day. Response options ranged from less than $1 \mathrm{~h}$ to 7 or more hours on a five-point scale.

Lifestyle changes were assessed using six questions on a seven-point Likert scale. These questions asked how participants' lifestyles may have changed over the past few months in categories such as focus, sleeping habits, eating habits, and amount of television watched. These were then averaged for an overall lifestyle change score and had an alpha of .80. Participants were then asked a series of four questions to assess their agreeableness to restrictions due to COVID-19. These questions were assessed on a sevenpoint Likert scale and included, "I believe we should be social (or physical) distancing," "I believe schools should open for the Fall 2020 semester," "I believe businesses should be forced to close," and "I believe we should be wearing masks." These four items were then averaged to create a score and had an alpha of .73 .

\section{COVID-19 Diagnosis Measures}

Participants were asked if they were previously diagnosed with COVID-19. They were able to respond with no; yes, I tested positive; yes, but I wasn't able to/did not get tested. All of the participants who answered either yes, I tested positive or yes, but I wasn't able to/did not get tested were combined and the variable was coded dichotomously for those with and without a history of COVID-19. Participants were also asked if they personally knew anyone that has had COVID-19 other than themselves (knowing someone who was diagnosed with COVID-19) where they could answer yes or no. This variable was also coded dichotomously. See Table 1 for descriptive statistics of all risk markers examined in the analyses, as well as supplemental Table 1 for correlations among all variables examined in the analyses.

\section{Analysis Plan}

Our goal was to identify potential risk markers for IPV perpetration among a national sample in the midst of the COVID-19 pandemic. Our outcome, IPV perpetration, was dichotomous, so we calculated unadjusted logistic regression analyses to identify the odds of IPV perpetration as a function of a one-unit increase on each predictor. One logistic regression was run per risk marker included in the study. Unadjusted odds ratios and $p$-values are reported for the expected change in the odds of IPV by each proposed predictor. We order the results presented below and in Table 2 in order of the magnitude of the effect size, starting with the predictor that had the largest effect size.

\section{Results}

\section{COVID-19 Related Risk Markers for IPV Perpetration}

The COVID-19 related risk markers that increased risk for IPV perpetration the most were located within mental health. A one-unit increase on our measure of feelings of loneliness 
Table 1 Descriptive statistics of risk markers examined in the study

\begin{tabular}{|c|c|c|c|c|c|}
\hline Variable & $M$ & $S D$ & Range & Alpha & $n(\%)$ \\
\hline Anxiety Symptoms & 1.92 & 0.82 & $1-4$ & .93 & - \\
\hline Depressive Symptoms & 13.58 & 11.88 & $0-50$ & .94 & - \\
\hline Substance Use & 0.56 & 0.79 & $0-4$ & .67 & - \\
\hline Feelings of Fear & 2.96 & 0.63 & $1-4$ & .86 & - \\
\hline Fear of getting COVID-19 & 4.38 & 1.73 & $1-7$ & - & - \\
\hline Perceived Stress & 2.58 & 0.85 & $1-5$ & .76 & - \\
\hline Feelings of Boredom & 2.65 & 1.00 & $1-5$ & .87 & - \\
\hline Perceived Well-Being & 6.29 & 2.08 & $0-10$ & .79 & - \\
\hline Feelings of Hopefulness & 5.28 & 1.10 & $1-8$ & .86 & - \\
\hline Feelings of Loneliness & 1.62 & 0.60 & $1-3$ & .83 & - \\
\hline Frequency of Going Outside for More than 15 Minutes & 3.46 & 1.25 & $1-5$ & - & - \\
\hline Amount of Time Spent Interacting with Friends/Family In Person & 1.84 & 1.53 & $1-8$ & - & - \\
\hline Amount of Time Spent Interacting with Friends/Family Virtually & 2.95 & 1.41 & $1-8$ & - & - \\
\hline Lifestyle Changes & 1.15 & 0.75 & $0-3$ & .80 & - \\
\hline Agreeable to Restrictions due to COVID-19 & 5.43 & 1.20 & $1-7$ & .73 & - \\
\hline Time Spent Reading/Watching the News & 1.86 & 0.81 & $1-5$ & - & - \\
\hline Time Spent on Social Media & 2.01 & 1.01 & $1-5$ & - & - \\
\hline Struggling Financially & 3.29 & 1.26 & $1-5$ & - & \\
\hline Suicidal Ideation & - & - & - & - & $146(40.1 \%)$ \\
\hline Working from Home & - & - & - & - & $110(30.1 \%)$ \\
\hline Has Been Furloughed since Mid-March 2020 & - & - & - & - & $18(4.9 \%)$ \\
\hline Has Been Fired since Mid-March 2020 & - & - & - & - & $23(6.3 \%)$ \\
\hline Working More Hours since Mid-March 2020 & - & - & - & - & $16(4.4 \%)$ \\
\hline Working Less Hours since Mid-March 2020 & - & - & - & - & $54(14.8 \%)$ \\
\hline Access to Same Financial Resources since pandemic began & - & - & - & - & $260(71.2 \%)$ \\
\hline Has Been Diagnosed with COVID-19 & - & - & - & - & $17(4.7 \%)$ \\
\hline Knowing Someone who was diagnosed with COVID-19 & - & - & - & - & $177(48.5 \%)$ \\
\hline
\end{tabular}

was significantly associated with a 5.33-fold increase in the odds of IPV perpetration $(O R=5.33, p<.001$; See Table 2). Next, a one-unit increase in anxiety symptoms was associated with a 2.75 -fold increase in IPV perpetration $(O R=2.75, p<.001)$. Further, greater perceived stress $(O R=2.71, p<.001)$, feelings of fear $(O R=2.61, p<.01)$, and feelings of boredom $(O R=2.41, p<.001)$ were each significantly linked with much higher odds of perpetrating IPV. Additionally, substance use $(O R=2.30, p<.001)$, lifestyle changes $(O R=2.08, p<.01)$, time spent on social media $(O R=1.92, p<.001)$, struggling financially $(O R=1.37$, $p<.05)$, fear of getting COVID-19 $(O R=1.27, p<.05)$, and depressive symptoms $(O R=1.08, p<.05)$ were all significant risk markers for IPV perpetration. Overall perceived well-being and feelings of hopefulness were each significant protective markers against IPV perpetration. More specifically, higher overall wellbeing was associated with a $29 \%$ decrease in the odds of participants reporting IPV perpetration $(O R=0.71, p<.001)$, whereas feelings of hopefulness was associated with a $34 \%$ decrease in the odds of participants reporting IPV perpetration $(O R=0.66, p<.05)$.
Time spent reading/watching the news, working from home, amount of time spent interacting with friends and family either virtually or in-person, frequency of going outside for more than $15 \mathrm{~min}$, and agreeableness to restrictions due to COVID-19 were not significant risk markers for IPV perpetration. Additionally, several salient COVID19 factors were not significant risk markers for IPV perpetration, including knowing someone who was diagnosed with COVID-19, job changes (new job, working more or less hours, since mid-March 2020, having been furloughed, having been fired), access to the same financial resources since the pandemic began, suicidal ideation, and having been diagnosed with COVID-19.

\section{Discussion}

This study found that the strongest risk markers for IPV perpetration during the COVID-19 pandemic were related to mental health and isolation factors, such as feelings of loneliness, anxiety symptoms, perceived stress, fear, boredom, 
Table 2 Unadjusted odds ratios for COVID-19 related risk markers for IPV perpetration

\begin{tabular}{|c|c|c|c|}
\hline Risk Marker & OR & $95 \% \mathrm{CI}$ & Type of Risk Marker \\
\hline Feelings of Loneliness & $5.33 * * *$ & {$[2.90,9.79]$} & Mental Health \& Isolation \\
\hline Anxiety Symptoms & $2.75 * * *$ & {$[1.82,4.16]$} & Mental Health \\
\hline Perceived Stress & $2.71 * * *$ & {$[1.69,4.36]$} & Mental Health \\
\hline Feelings of Fear & $2.61 * *$ & {$[1.40,4.86]$} & Mental Health \\
\hline Feelings of Boredom & $2.41 * * *$ & {$[1.63,3.58]$} & Mental Health \\
\hline Substance Use & $2.30 * * *$ & {$[1.61,3.29]$} & Mental Health \\
\hline Lifestyle Changes & $2.08 * *$ & {$[1.32,3.28]$} & Lifestyle \\
\hline Time Spent on Social Media & $1.92 * * *$ & {$[1.40,2.62]$} & Lifestyle \\
\hline Time Spent Reading/Watching the News & 1.46 & {$[0.99,2.14]$} & Lifestyle \\
\hline Working from Home & 1.44 & {$[0.63,3.29]$} & Lifestyle \\
\hline Struggling Financially & $1.37 *$ & {$[1.04,1.82]$} & Financial \\
\hline Fear of Getting COVID-19 & $1.27 *$ & {$[1.02,1.59]$} & Mental Health \\
\hline Amount of Time Spent Interacting with Friends/Family Virtually & 1.17 & {$[0.88,1.41]$} & Isolation \\
\hline Amount of Time Spent Interacting with Friends/Family In Person & 1.11 & {$[0.97,1.40]$} & Isolation \\
\hline Depressive Symptoms & $1.08 *$ & {$[1.05,1.11]$} & Mental Health \\
\hline Frequency of Going Outside for More than 15 Minutes & 1.07 & {$[0.80,1.42]$} & Isolation \\
\hline Agreeable to Restrictions due to COVID-19 & 1.00 & {$[0.75,1.35]$} & Lifestyle \\
\hline Knowing Someone who was diagnosed with COVID-19 & 0.83 & {$[0.41,1.69]$} & COVID-19 Diagnosis \\
\hline Working Less Hours since Mid-March 2020 & 0.78 & {$[0.31,1.98]$} & Financial \\
\hline Access to Same Financial Resources since pandemic began & 0.72 & {$[0.34,1.72]$} & Financial \\
\hline Working More Hours since Mid-March 2020 & 0.71 & {$[0.16,3.27]$} & Financial \\
\hline Perceived Well-Being & $0.71 * * *$ & {$[0.59,0.84]$} & Mental Health \\
\hline Feelings of Hopefulness & $0.66 *$ & {$[0.48,0.91]$} & Mental Health \\
\hline Suicidal Ideation & 0.65 & {$[0.32,1.32]$} & Mental Health \\
\hline Has Been Furloughed since Mid-March 2020 & 0.49 & {$[0.14,1.80]$} & Financial \\
\hline Has Been Fired since Mid-March 2020 & 0.46 & {$[0.15,1.44]$} & Financial \\
\hline Has Been Diagnosed with COVID-19 & 0.31 & {$[0.10,1.01]$} & COVID-19 Diagnosis \\
\hline
\end{tabular}

Note: $\mathrm{OR}=$ Odds Ratio $\mathrm{CI}=$ Confidence Interval

$* p<.05 . * * p<.01 . * * * p<.001$ (two-tailed)

and substance use. Although these risk markers could also occur outside of the COVID-19 pandemic, they are potentially exacerbated by the social isolation related to the pandemic (WHO, 2020c). The strongest risk marker by far for IPV perpetration was loneliness, which increased the likelihood of a participant reporting perpetrating IPV by over $400 \%$. Using the ABC-X model (Boss, Bryant, \&Mancini, 2016) to interpret this result, loneliness can be viewed as a lack of connection or social support, and support is considered a resource that promotes healthy coping when faced with a stressful event. The lack of support from others may make coping with stress more difficult, thus increasing the likelihood that someone resorts to violence. When assessing for IPV perpetration, examining social networks and feelings of loneliness may aid in the identification of those at risk for perpetrating IPV against their partners during the stressful time of the pandemic. Interestingly, loneliness has not been a widely examined risk marker for IPV perpetration in the literature outside of being a construct for depressive symptoms (e.g., Johnson et al., 2015). Future examination of loneliness as a potent risk marker for IPV perpetration even outside of the COVID-19 pandemic may be warranted, but clearly, during the pandemic it seems highly relevant.

Other mental health factors were significant risk markers for IPV perpetration (e.g., anxiety, stress, fear, boredom, substance use, depressive symptoms). This is important for helping professionals, as it can be a means of assessment and intervention efforts in combating IPV perpetration among men and women. Poor mental health outcomes have been linked to IPV perpetration in prior research (Author et al., 2019; Cafferky et al., 2018; Oram et al., 2014; Shorey et al., 2012), so this may not be a surprising finding. However these results still warrant careful attention due to the increased mental health struggles associated with the social isolation during the COVID-19 pandemic (Wang et al., 2020; WHO, 2020b; WHO, 2020c). Negative mental health symptoms may or may not be an outcome related to COVID-19, but since it is known that mental health problems have increased 
due to the COVID-19 pandemic, it is important to be aware of the link between mental health problems and IPV perpetration. Additionally, fear related specifically to contracting COVID-19 was a risk marker for IPV perpetration, which may be an important aspect of mental health to assess during this time. Overall, given the increased strain the pandemic has placed on mental health, and the elevated risk of IPV with greater mental health challenges, it is important for helping professionals to assess for mental health, such as anxiety, stress, fear, boredom, depression, and most importantly, loneliness.

Lifestyle changes (measured by an increased difficulty to focus, watching more television, eating more, changes in bedtime routines, and getting less sleep since March 2020) was also related to IPV perpetration. This risk marker may be easier to link directly to the COVID-19 pandemic, as it specifically asked about changes during the time of the pandemic. Reported lifestyle changes increased the likelihood of a participant reporting IPV perpetration by over two times. The lifestyle changes examined in the study, such as sleeping disturbances, have been linked to poor mental health outcomes (e.g., Zochill \& Thorsteinsson, 2018). Negative lifestyle changes may serve as a useful assessment point, as individuals may feel less threatened to answering questions about their current lifestyle changes they have experienced since the COVID-19 pandemic, as there remains a stigma or embarrassment for some individuals regarding mental health (Bathje \& Pryor, 2011; Gulliver et al., 2010).

There was a $37 \%$ increase in the likelihood of participants reporting have perpetrated IPV against their partner if they reported struggling financially. Although not the strongest risk marker examined, it may still be a useful risk marker to aid in identification of those at risk for IPV perpetration. Struggling financially may be viewed as a lack of a viable resource, and available resources can impact how someone reacts to a stressful event (Hill, 1958). Struggling financially has been related to other risk markers for IPV perpetration, such as mental health issues, physical health problems, and relationship conflict/distress (Dakin and Wampler, 2008; Stronks et al., 1998). Financial struggles have been related to increased couple arguments centered around finances, as well as hostile interactions (Britt et al., 2010; Conger et al., 1990). With many individuals experiencing economic hardship during the COVID-19 pandemic (International Labor Organization, 2020), and families reporting loss of job or financial insecurity as a source of family stress (Carroll et al., 2020), it is important to examine financial insecurity in assessment and intervention efforts related to reduction in IPV.

It is also important to focus on what factors were related to a decreased likelihood of perpetrating physical IPV. We found that perception of well-being and feelings of hopefulness were significant protective markers, meaning that they were associated with the reduction of reporting IPV perpetration. This is an important finding, because it provides a potential resource to target for preventing and potentially treating IPV perpetration. For helping professionals with an extended relationship with clients, it may be useful to use strength-based approaches or tenets of positive psychology to aid in feelings of hopefulness and well-being among clients (Carr, 2011; Gander et al., 2013; Slade, 2010).

The insignificant results of this study are also of interest. A variety of the factors related to lifestyle changes and isolation were found insignificant (e.g., working from home, the amount of time interacting with friends/family either virtually or in-person, and amount of time spent outside). It may be that there is greater variability in how individuals reacted to these lifestyle changes, or their perception of these events. For example, some people may very much dislike working from home, while others feel as though they are thriving in their new work environment. Additionally, since loneliness was the strongest risk marker for IPV perpetration that we examined, and many risk markers related to isolation were not, it highlights the importance in examining how stressors are perceived by the individual. Participants identified that they were experiencing feelings of loneliness, whereas spending more time alone does not necessarily mean someone perceives being more isolated as feeling lonely. It has also been pointed out that a probable outcome of the COVID-19 pandemic could be increased resiliency (PeConga et al., 2020), which could also be linked to varying outcomes related to lifestyle changes during the pandemic. It may also be important to note that we did not collect data on these lifestyle factors prior to the COVID-19 pandemic, it is also possible that for some people, these may not have actually been big lifestyle changes (e.g., someone may not have gone outside very often prior to the stay-at-home orders, so this remained stable during the pandemic).

Surprisingly to us, several of the financial stress-related risk markers were found to be insignificant risk markers for IPV perpetration (e.g., having been furloughed, fired, working more hours, working less hours, changing jobs, and having similar access to resources). When examining the data, these factors had relatively large confidence intervals, suggesting that individuals may react much differently from one another in these situations. For some, financial stress or unemployment may create hostile environments that increase conflict (Britt et al., 2010; Conger et al., 1990). Whereas for others, one's job may be a huge source of stress in their lives (American Psychological Association [APA], 2015; Nixon et al., 2011), and losing a job may lead to positive changes. Another possibility is that someone may have lost a job, but are receiving unemployment benefits and do not interpret or perceive the event as stressful. This highlights the component of the ABC-X model that focuses on how stressful events are interpreted, and how access to resources 
during the stressful event can impact a person's perception of the event. Some factors we examined, such as working less hours or changing jobs, may have had a positive impact on some individuals. Again, we hypothesize that the wide variability in the $95 \%$ confidence intervals may be due to how these financial/work-related life changes were perceived or interpreted by the individual, where work was presumably a source of strength for some and a source of hardship for others. Collecting more nuanced or contextualized data may be helpful to examine the process on how these potential risk markers may relate to IPV perpetration.

\section{Limitations and Future Research}

A main limitation of this research is that the data is crosssectional, and although the data were collected during the COVID-19 pandemic, we were unable to examine how these risk markers for IPV perpetration changed from preCOVID-19 to during the pandemic. Additionally, cross-sectional data only allows us to report on relationships between variables, and we cannot say the risk markers examined lead to IPV perpetration. Another limitation to the study is the lack of diversity of the sample (e.g., predominately White with a 4-year college degree), which limits the generalizability of the results. Future research using larger and more diverse samples could further extent the generalizability of the results. Additionally, our study examined risk markers for IPV perpetration for both men and women, which did not allow for the examination of gender differences. Future research should consider exploring potential gender differences in risk markers for IPV perpetration and victimization related to COVID-19.

Another strong limitation of the study was that participants were also asked to identify incidents of IPV perpetration within the past year, when the pandemic had only been occurring for about five months. Thus, we cannot rule out that some of these reported incidences of IPV occurred before the primary onset of COVID-19. This is something to be considered for future research studies. Additionally, this study examined physical, psychological, and sexual IPV as one measure, future research may benefit from examining the types of IPV perpetration separately in order to gain a more nuanced understanding of how risk markers relate to IPV. We also chose to dichotomize the outcome variable of IPV perpetration. This limits our ability to examine frequency or severity of the IPV perpetrated. Future research should take into account if certain risk markers for IPV perpetration are related to more frequent or severe acts of IPV perpetration. Lastly, it is important to note that there are other pertinent factors related to COVID-19 (e.g., lack of access to IPV prevention education and services) that were not examined in this study that would be beneficial to include in future research.

\section{Conclusion}

Although a considerable amount of attention and concern has been given to the increase in IPV rates since the COVID19 pandemic and consequential lockdown and stay-at-home orders, this is the first study to our knowledge that examines risk markers for IPV perpetration related to COVID-19. Examining risk markers is an important tool in aiding in assessment, identification, and intervention efforts to reduce IPV. This study found that the strongest risk markers for IPV in a sample surveyed during the COVID-19 pandemic were related to isolation and mental health challenges, such as loneliness, anxiety, stress, fear, boredom and substance use. Additionally, lifestyle changes since March 2020 and fear of contracting COVID-19 were also significant risk markers. Perceived well-being and feelings of hopefulness were protective markers against IPV perpetration during the time of the COVID-19 pandemic. Examining COVID-19 related risk markers may be useful for helping professionals to help assess for IPV perpetration during this time of heightened attention to IPV perpetration.

\section{Declarations}

Conflict of Interest The authors declare that they have no conflict of interest.

\section{References}

Author et al., 2019.

Author et al., 2020.

Bathje, G., \& Pryor, J. (2011). The relationships of public and selfstigma to seeking mental health services. Journal of Mental Health Counseling, 33(2), 161-176.

Bendau, A., Petzold, M. B., Pyrkosch, L., Maricic, L. M., Betzler, F., Rogoll, J., et al. (2020). Associations between COVID-19 related media consumption and symptoms of anxiety, depression and COVID-19 related fear in the general population in Germany. European Archives of Psychiatry and Clinical Neuroscience, 721(2), 283-291.

Benson, T., Sladen, J., Liles, A., \& Potts, H. W. W. (2019). Personal wellbeing score (PWS)- a short version of ONS4: Development and validation in social prescribing. BMJ Open Quality, 8, 1-9.

Boss, P., Bryant, C. M., \& Mancini, J. A. (2016). Family stress management: A contextual approach. Sage Publications.

Breiding, M. J., Chen, J., \& Black, M. C. (2014). Intimate partner violence in the United States - 2010. Atlanta, GA: National Center for Injury Prevention and Control, Centers for Disease Control and Prevention. Retrieved from https://www.cdc.gov/ violenceprevention/pdf/cdc_nisvs_ipv_report_2013_v17_single_a.pdf. 
Britt, S. L., Huston, S., \& Durband, D. (2010). The determinants of money arguments between spouses. Journal of Financial Therapy, $42-60$.

Bureau of Labor Statistics. (2020). The Employment Situation: April 2020. Department of Labor, USA. USDL-20-0815.

Cafferky, B. M., Mendez, M., Anderson, J. R., \& Stith, S. M. (2018). Substance use and intimate partner violence: A meta-analytic review. Psychology of Violence, 8(1), 110-132.

Campbell, J. C. (2002). Health consequences of intimate partner violence. The Lancet, 359(9314), 1331-1336.

Carfì, A., Bernabei, R., \& Landi, F. (2020). Persistent symptoms in patients after acute COVID-19. JAMA, 324(6), 603-605.

Carr, A. (2011). Positive psychology: The science of happiness and human strengths. Routledge.

Carroll, N., Sadowski, A., Laila, A., Hruska, V., Nixon, M., Ma, D. W., \& Haines, J. (2020). The impact of COVID-19 on health behavior, stress, financial and food security among middle to high income Canadian families with young children. Nutrients, 12(8), 2352.

Chapman University. (2018). The Chapman University survey of American fears, wave 5. Earl Babbie Research Center.

City of San Antonio. (2020). Social distancing doesn't mean safety distancing. City of San Antonio Department of Government and Public Affairs. Retrieved from https://www.sanantonio.gov/gpa/ News/ArtMID/24373/ArticleID/18724/Social-distancing-doesn

Cohen, S., Kamarck, T., \& Mermelstein, R. (1983). A global measure of perceived stress. Journal of Health and Social Behavior, 24, 385-396.

Coker, A. L., Smith, P. H., Bethea, L., King, M. R., \& McKeown, R. E. (2000). Physical health consequences of physical and psychological intimate partner violence. Archives of Family Medicine, 9(5), 451

Conger, R. D., Elder, G. H., Lorenz, F. O., Conger, K. J., Simons, R. L., Whitbeck, L. B., Huck, S., \& Melby, J. N. (1990). Linking economic hardship to marital quality and instability. Journal of Marriage and Family, 52, 643-656.

Crandall, C. S., \& Moriarty, D. (1995). Physical illness stigma and social rejection. British Journal of Social Psychology, 34(1), 67-83.

Dakin, J., \& Wampler, R. (2008). Money doesn't buy happiness, but it helps: Marital satisfaction, psychological distress, and demographic differences between low- and middle-income clinic couples. American Journal of Family Therapy, 36, 300-311.

Docherty, A. B., Harrison, E. M., Green, C. A., Hardwick, H. E., Pius, R., Norman, L., ... \& Semple, M. G. (2020). Features of 20133 UK patients in hospital with covid-19 using the ISARIC WHO clinical characterisation protocol: Prospective observational cohort study. BMJ, 369. doi: https://doi.org/10.1136/bmj.m1985.

Dutton, M. A., Mitchell, B., \& Haywood, Y. (1996). The emergency department as a violence prevention center. Journal of the American Medical Women's Association (1972), 51(3), 92-95.

Egede, L. E. (2007). Major depression in individuals with chronic medical disorders: Prevalence, correlates and association with health resource utilization, lost productivity and functional disability. General Hospital Psychiatry, 29(5), 409-416.

Fitzpatrick, K. M., Harris, C., \& Drawve, G. (2020). Fear of COVID-19 and the mental health consequences in America. Psychological trauma: Theory, research, practice, and policy, 12(S1), S17-S21).

Gander, F., Proyer, R. T., Ruch, W., \& Wyss, T. (2013). Strengthbased positive interventions: Further evidence for their potential in enhancing well-being and alleviating depression. Journal of Happiness Studies, 14(4), 1241-1259.

Gulliver, A., Griffiths, K. M., \& Christensen, H. (2010). Perceived barriers and facilitators to mental health help-seeking in young people: A systematic review. BMC Psychiatry, 10(1), 113.

Hill, R. (1958). 1. Generic features of families under stress. Social Casework, 39(2-3), 139-150.
International Labor Organization . (2020). How will COVID-19 affect the world of work? Retrieved from: https://www.ilo.org/global/ topics/coronavirus/lang-en/index.htm

Hughes, M. E., Waite, L. J., Hawkley, L. C., \& Cacioppo, J. T. (2004). A short scale for measuring loneliness in large surveys: Results from two population-based studies. Research on Aging, 26(6), $655-672$.

James, S. E., Johnson, J., \& Raghavan, C. (2004). "I couldn't go anywhere": Contextualizing violence and drug abuse: A social network study. Violence Against Women, 10, 991-1014.

Jefferson County Sheriff's Office. (2020). Money J. Jefferson County experiencing increase in domestic violence crimes during Covid19 pandemic. Retrieved from: https://jeffcosheriffal.com

Johnson, W. L., Giordano, P. C., Manning, W. D., \& Longmore, M. A. (2015). The age-IPV curve: Changes in the perpetration of intimate partner violence during adolescence and young adulthood. Journal of Youth and Adolescence, 44(3), 708-726.

Kaukinen, C. (2020). When stay-at-home orders leave victims unsafe at home: Exploring the risk and consequences of intimate partner violence during the COVID-19 pandemic. American Journal of Criminal Justice, 1-12.

McKee-Ryan, F., Song, Z., Wanberg, C. R., \& Kinicki, A. J. (2005). Psychological and physical well-being during unemployment: A meta-analytic study. Journal of Applied Psychology, 90(1), 53.

Moreira, D. N., \& da Costa, M. P. (2020). The impact of the Covid-19 pandemic in the precipitation of intimate partner violence. International Journal of Law and Psychiatry, 71, 101606.

NIDA. (2012). Resource guide: Screening for drug use in general medical settings. Retrieved from https://archives.drugabuse.gov/publi cations/resource-guide-screening-drug-use-in-general-medicalsettings on 2021, January 5.

Nixon, A. E., Mazzola, J. J., Bauer, J., Krueger, J. R., \& Spector, P. E. (2011). Can work make you sick? A meta-analysis of the relationships between job stressors and physical symptoms. Work \& Stress, 25(1), 1-22.

Olsen, L. R., Jensen, D. V., Noerholm, V., Martiny, K., \& Bech, P. (2003). The internal and external validity of the major depression inventory in measuring severity of depressive states. Psychological Medicine, 33, 351-356.

Osman, A., Bagge, C. L., Guitierrez, P. M., Konick, L. C., Kooper, B. A., \& Barrios, F. X. (2001). The suicidal behaviors questionnairerevised (SBQ-R): Validation with clinical and nonclinical samples. Assessment, 5, 443-454.

Oram, S., Trevillion, K., Khalifeh, H., Feder, G., \& Howard, L. M. (2014). Systematic review and meta-analysis of psychiatric disorder and the perpetration of partner violence. Epidemiology and Psychiatric Sciences, 23, 361-376.

Peterman, A., Potts, A., O’Donnell, M., Thompson, K., Shah, N., Oertelt-Prigione, S., \& van Gelder, N. (2020). Pandemics and violence against women and children. Center for Global Development Working Paper, 528.

Rajkumar, R. P. (2020). COVID-19 and mental health: A review of the existing literature. Asian Journal of Psychiatry, 102066.

Schultz, A. B., Chen, C. Y., \& Edington, D. W. (2009). The cost and impact of health conditions on presenteeism to employers. Pharmacoeconomics, 27(5), 365-378.

Shorey, R. C., Elmquist, J., Ninnemann, A., Brasfield, H., Febres, J., Rothman, E. F., et al. (2012). The association between intimate partner violence perpetration, victimization, and mental health among women arrested for domestic violence. Partner Abuse, $3(1), 3-21$

Slade, M. (2010). Mental illness and well-being: The central importance of positive psychology and recovery approaches. BMC Health Services Research, 10(1), 1-14.

Smith, S. G., Basile, K. C., Gilbert, L. K., Merrick, M. T., Patel, N., Walling, M. et al. (2017). National intimate partner and sexual 
violence survey (NISVS): 2010-2012 State report. Retrieved from https://www.cdc.gov/violenceprevention/pdf/nisvs-staterepor tbook.pdf

Snyder, C. R., Harris, C., Anderson, J. R., Holleran, S. A., Irving, L. M., Sigmon, S. T., et al. (1991). The will and the ways: Development and validation of an individual-differences measure of hope. Journal of Personality and Social Psychology, 60, 570-585.

Spitzer, R. L., Kroenke, K., Williams, J. B. W., \& Lowe, B. (2006). A brief measure for assessing generalized anxiety disorder. Arch Inern Med., 166, 1092-1097.

Stronks, K., van de Mheen, H. D., \& Mackenbach, J. P. (1998). A higher prevalence of health problems in low-income groups: Does it reflect relative deprivation? Journal of Epidemiology \& Community Health, 52(9), 548-557.

Struk, A. A., Carriere, J. S. A., Cheyne, J. A., \& Danckert, J. (2017). A short boredom proneness scale: Development and psychometric properties. Assessment, 24(3), 346-359.

van Gelder, N., Peterman, A., Potts, A., O’Donnell, M., Thompson, K., Shah, N., \& Oertelt-Prigione, S. (2020). COVID-19: Reducing the risk of infection might increase the risk of intimate partner violence. EClinicalMedicine. Advanced online publication. doi: https://doi.org/10.1016/j.eclinm.2020.100348.

Wang, C., Pan, R., Wan, X., Tan, Y., Xu, L., Ho, C. S., \& Ho, R. C. (2020). Immediate psychological responses and associated factors during the initial stage of the 2019 coronavirus disease
(COVID-19) epidemic among the general population in China. International Journal of Environmental Research and Public Health, 17(5), 1729.

Wong, J., \& Mellor, D. (2014). Intimate partner violence and women's health and wellbeing: Impacts, risk factors and responses. Contemporary Nurse, 46(2), 170-179.

World Health Organization. (2020a). COVID-19 and violence against women: What health sector/system can do. Retrieved from: https:// apps.who.int/iris/bitstream/handle/10665/331699/WHO-SRH-20. 04-eng.pdf

World Health Organization. (2020b). Mental health and psychosocial considerations during the COVID-19 outbreak. Retrieved from:. https://www.who.int/ docs/default-source/coronaviruse/mentalhealth-considerations.pdf.

World Health Organization. (2020c). Mental health and COVID-19. Retrieved from: http://www.euro.who.int/en/health-topics/healthemergencies/coronavirus-covid-19/novel-coronavirus-2019-ncovtechnical-guidance/ coronavirus-disease-covid-19-outbreak-technical-guidance-europe/ mental-health-and-covid-19.

Zochil, M. L., \& Thorsteinsson, E. B. (2018). Exploring poor sleep, mental health, and help-seeking intention in university students. Australian Journal of Psychology, 70(1), 41-47.

Publisher's Note Springer Nature remains neutral with regard to jurisdictional claims in published maps and institutional affiliations. 